\title{
Los estudiantes de Comunicación en las redes sociales: estudio comparativo entre Brasil, Colombia y España
}

\section{Communication students in social media: Comparative study among Brazil, Colombia and Spain}

\author{
Santiago TEJEDOR ${ }^{1}$ (D) 0000-0002-5539-9800 \\ Ricardo CARNIEL BUGS' (id) 0000-0002-9303-9515 \\ Santiago GIRALDO LUQUE1 (D) 0000-0003-0024-7081
}

\section{Resumen}

El estudio de las redes sociales ha generado importantes datos y reflexiones sobre la concepción y el tipo de utilización que los jóvenes hacen de las plataformas digitales online. Sin embargo, se plantea la necesidad de trabajos que analicen el público joven desde una mayor segmentación y especificación de perfiles. Este artículo presenta los resultados de una investigación realizada en Brasil, Colombia y España, con una muestra integrada exclusivamente por estudiantes universitarios de Comunicación. El trabajo analiza cómo estos alumnos (y futuros comunicadores) valoran y utilizan las redes sociales y qué importancia les confieren en su formación y en su futuro profesional. A través de una metodología de trabajo híbrida basada en una encuesta matriz con preguntas cuantitativas y abiertas, el estudio concluye que los estudiantes de Comunicación valoran de forma muy positiva las redes sociales y las conciben como un fenómeno consolidado, con una larga proyección en el tiempo y una gran utilidad para informarse o contactar con profesionales. No obstante, se observa por parte del alumnado una preocupante pérdida de reconocimiento y de credibilidad hacia el trabajo de los periodistas en las redes y, de forma más aguda, hacia los políticos y fuentes gubernamentales.

Palabras clave: Comunicación. Internet. Medios sociales. Periodismo. Universitarios.

\begin{abstract}
Research on social media has generated important data and reflections on the conception and the type of use young users make of the digital online platforms. However, studies that analyze young people from a greater profile segmentation and specification are required. This article presents the results of a research carried out in Brazil, Colombia and Spain, with a sample composed exclusively of university students from the field of communication. The study focuses on analyzing how students (and future communicators) value and use social media and how important these tools are for their training and professional future. A hybrid methodology based on a matrix survey with quantitative and open questions was used. It may be concluded that communication students value social

1 Universitat Autònoma de Barcelona, Facultat de Ciències de la Comunicación, Departamento de Periodismo y Ciencias de la Comunicación. Carrer de las Vinyas, 08193, Bellaterra (Cerdanyola del Vallès), Barcelona, España. Correspondencia para/Correspondence to: R. CARNIEL BUGS. E-mail: <ricardo.carniel@uab.cat>.

Recibido el 10 del agosto del 2017 y aprobado el 15 septiembre del 2017.
\end{abstract}

Como citar este articulo/How to cite this article

Tejedor S.; Carniel Bugs R.; Giraldo Luque S. Los estudiantes de Comunicación en las redes sociales: estudio comparativo entre Brasil, Colombia y España. Transinformação, v. 30, n. 2, p. 267-276, 2018. http://dx.doi.org/10.1590/2318-08892018000200010 
media positively and conceive them as a consolidated and long-lasting phenomenon, as well as a useful mean to obtain information or contact professionals. Nonetheless, it was observed that the students are concerned about the loss of recognition and credibility of journalists in social media and, more specifically, of politicians and government sources.

Keywords: Communication. Internet. Social media. Journalism. University students.

\section{Introducción}

El consumo de noticias se ha convertido en una experiencia social. Las redes sociales y los blogs, especialmente, han contribuido a este cambio (Purcell et al., 2010). Estas plataformas han impulsado una transformación de los contenidos informativos mediante la generación de piezas que destacan por ser "portátiles", "personalizadas" y "participativas" (Matsa; Mitchell, 2014). De este modo, asistimos a un conjunto de cambios que afectan a las rutinas de producción periodística desde la concepción del mensaje hasta su producción, distribución y recepción. No obstante, estudios como los de Masip et al. (2015) apuntan que un porcentaje elevado de la ciudadanía sigue optando por los medios de información "más tradicionales" para acceder a contenidos informativos "de confianza". Esta tendencia se detecta igualmente entre los periodistas. A pesar de que estos hacen un uso amplio de las redes sociales, les conceden una menor credibilidad que a los medios tradicionales (Herrero Curiel, 2015).

Se han detectado una gran variedad de perfiles entre los usuarios de las redes sociales que van desde el nativo tecnológico, el inmigrante tecnológico, el reflexivo tecnológico o el escéptico tecnológico (Ruano; Congote; Torres, 2016). También ha sido de interés esclarecer las motivaciones que llevan a los internautas a crear perfiles y utilizar dichas plataformas. Así, el "para qué" (Baek et al., 2011) y el "por qué" (Whiting; Williams, 2013) se han convertido en uno de los principales objetos de estudio dentro de esta temática. Bulut y Doğan (2017) proponen una clasificación de los usos de las redes sociales a partir de siete motivaciones específicas. Una de ellas hace referencia a la utilización de las redes sociales con fines empresariales o laborales. Los autores diferencian, dentro de ella, el uso de las redes para promocionar un trabajo específico (Baek et al., 2011) o para dar a conocer sus avances profesionales y proyectar su perfil digital dentro de un escenario laboral (Papacharissi; Mendelson, 2011).

Desde hace más de una década, estudios alertaban de la necesidad de investigar de forma continuada los hábitos de uso y consumo de los jóvenes en el ciberespacio (Muñoz-Rivas; Navarro; Ortega, 2003; Carbonell et al., 2012). Más allá de los estudios que ofrecen una visión agorera sobre el uso de Internet por este perfil de usuarios, Ruiz de Miguel (2016) indica que los jóvenes aprovechan las posibilidades ofrecidas por los nuevos dispositivos digitales, sin caer en una utilización patológica. El estudio sobre el perfil y tipo de uso que los universitarios españoles hacen del teléfono móvil y de internet concluye que el móvil es un instrumento cotidiano para los jóvenes, pero no se detecta un uso abusivo del mismo ni "una relación peligrosa" con estos aparatos (Ruiz De Miguel, 2016). No obstante, dos años antes, Cáceres y Morales (2014) estudiaron una experiencia de desconexión de los medios durante 24 horas por estudiantes universitarios, detectando un claro crecimiento de la comunicación mediada frente a la presencial, y señalan la existencia de una actitud de "hiperconexión" en estos jóvenes, hasta el punto que la ausencia de conectividad puede generarles malestar.

Por otro lado, estudios han destacado la existencia de una relación entre el uso de internet y una mayor implicación política de los jóvenes tanto en escenarios presenciales como virtuales (Espinar-Ruiz; González-Río, 2015). Otras investigaciones identificaron diferencias en el uso de internet según el tipo de disciplina de los estudiantes, estableciendo que la valoración de las tecnologías de la información y la comunicación variaba según el área de estudios. Alumnos de biología, matemáticas o ingeniería, entre otros, consideraban estas herramientas como "recursos fundamentales" en sus actividades académicas y en su vida en general. Ya los de ciencias sociales las concebían como "instrumentos de socialización"; mientras que los de humanidades y artes las identificaban como "herramientas secundarias", tanto en el ámbito académico como en el de la socialización (Crovi; López, 2012). Estas transformaciones demandan una redefinición del perfil del comunicador (Salaverría, 2016) y, por ende, una reflexión 
sobre las habilidades y competencias que contemplan los planes de estudio del ámbito de la comunicación (Pérez Tornero; Tejedor, 2016).

El estudio comparativo (Brasil, Colombia y España) sobre la concepción y el nivel de uso de las redes sociales permite, en este sentido, reformular parámetros decisivos para el estudio de las redes, como plataformas comunicativas, por un lado; y para el análisis de su importancia entre los usuarios más jóvenes, por otro. Asimismo, plantea las particularidades derivadas de nichos específicos de usuarios, como el alumnado de comunicación.

El artículo responde a la siguiente pregunta de investigación de ámbito descriptivo: ¿Qué tipo de uso y que valoración poseen los estudiantes de Comunicación de Brasil, Colombia y España - y futuros periodistas - de las redes sociales?". Con relación a ella, en el marco del trabajo, se han considerado las siguientes hipótesis:

- H1: Los estudiantes de Comunicación de estos países presentan una concepción común de las redes sociales en cuanto a su definición, a su importancia y a su utilidad, más allá de las diferencias socioeconómicas que existen entre estos tres países.

- H2: El alumnado de Comunicación identifica las redes sociales con cualidades y características positivas, obviando los elementos negativos.

- H3: Los alumnos no valoran las posibilidades de desarrollo profesional que les ofrecen las redes sociales.

\section{Escenarios del estudio: Brasil, Colombia y España}

La investigación ha seleccionado tres países que se destacan por la amplia presencia de internet y, especialmente, por el crecimiento de la Red entre los usuarios más jóvenes. Por un lado, Brasil se ha convertido en uno de los mayores ecosistemas mediáticos iberoamericanos: más de 10.400 emisoras de radio y 550 emisoras de TV - comerciales, públicas o comunitárias - y más de 20 mil retransmissoras de TV, sin contar las licencias de televisión digital (Brasil, 2017). Por otro lado, la Associação Nacional de Jornais (2015) contabiliza unos 5.219 periódicos - 784 de circulación diaria. Así, Brasil se consolida como el cuarto país del mundo en número de cabeceras y el octavo en cantidad de periódicos en circulación. Los datos indican un periódico por cada 38.852 habitantes, y las ediciones digitales de los diarios han doblado sus cifras, ocupando un 11,4\% de la circulación general. Además, se ha experimentado una expansión del 118,0\% (500.370 frente a las 228.944 del año anterior). A ello se une un incremento del acceso a las cabeceras informativas digitales desde smartphones. Entre enero y diciembre de 2014, el acceso por esta vía saltó del 13,0\% al 27,0\% del total. Internet es el segundo medio, después de la televisión, que más utilizan los brasileños para informarse. El 26,0\% de los entrevistados escogieron Internet como primera opción, mientras que el 49,0\% situaron la Red como una de sus dos primeras opciones (Instituto Brasileiro de Opinião Publica e Estatística, 2016). Asimismo, otro estudio contabiliza 139 millones de cibernautas, con más de 122 millones "internautas activos" en redes sociales (58,0\% de la población). El país ya es el mayor usuario de redes sociales de América Latina; un internauta invierte una media diaria de ocho horas y 56 minutos en internet, de las cuales tres horas y 43 minutos se centran en redes sociales (Kemp, 2017).

Colombia, por su parte, está entre los países latinoamericanos con un mayor crecimiento de los servicios de telefonía móvil, por un lado, y de iniciativas de alfabetización digital, por otro (Unión Internacional de Telecomunicaciones, 2012). La ciudad de Medellín, por ejemplo, posee más de 560 puntos de conexión gratuita a internet (Medellín..., 2015). El país se ha consolidado como líder en conectividad en América Latina y ocupa el puesto 27 en el Live Stats de 2016. Colombia acumula proyectos y reconocimientos vinculados con las tecnologías de la comunicación y la digitalización, como el premio City of the Year en 2013 a la ciudad de Medellín, o iniciativas como Medellín Ciudad Inteligente o Antioquia Digital (Gobernación de Antioquia, 2013), Plan Vive Digital, entre otros. Desde 2014, existen más de 25 millones de personas conectadas a internet y más de 20 millones de usuarios de Facebook, 25\% de los cuales poseen entre 15 y 24 años de edad (Colombia, 2014). 
España cuenta con una penetración de Internet del 82\% y se observa un incremento de los usuarios (pasando de 35,7 millones en 2015 a 37,87 millones en 2017). Las redes sociales también multiplicaron sus seguidores, alcanzando los 25 millones en 2017, por lo que su penetración se sitúa en un 54\% (Kemp, 2017). Según el “Estudio Anual de Redes Sociales 2017" de Interactive Advertising Bureau - Spain y Elogia (2017), Facebook tiene el mayor número de usuarios en el país (91\%), seguida de WhatsApp (89\%), YouTube (71\%) y Twitter (50\%).

\section{Procedimientos metodológicos}

Este estudio es parte de la última fase de un proyecto de investigación iniciado en el curso 2013-2014, para realizar un análisis diagnóstico sobre cómo los estudiantes de comunicación de Brasil, Colombia y España conciben, valoran y utilizan las redes sociales en los procesos de acceso, consulta y producción de contenidos digitales online. La investigación, focalizada en el curso 2016-2017, ha contado con la participación de un centenar de estudiantes de cada país (de ambos sexos, aunque en su mayoría mujeres; con edades entre 18 y 23 años). El porcentaje de hombres y mujeres que han participado en el estudio ha sido muy similar en cada país: Brasil (30,8\% hombres; $69,2 \%$ mujeres), Colombia (34,1\% y 65,2\%, respectivamente) y España (38,3\% y 61,3\%, respectivamente). La muestra, concebida como el conjunto de elementos de la población a los que se pide que participen en la investigación, corresponde a tres países con una amplia inversión en iniciativas vinculadas con las tecnologías de la información y un incremento en sus respectivos niveles de acceso a internet. En cada país, una universidad que impartiese estudios de Comunicación fue seleccionada a partir de los principales rankings o directorios internacionales de clasificación de los centros universitarios en Iberoamérica.

La investigación ha adoptado una metodología de trabajo híbrida basada en una encuesta matriz que cruza preguntas de tipo cuantitativo con cuestiones abiertas, permitiendo un trabajo más cualitativo en la lectura e interpretación de las respuestas. Las variables principales de las preguntas han sido las siguientes: (a) Concepción de una red social (en su desempeño profesional y en su tiempo de ocio); (b) Valoración de las opciones profesionales que, a su juicio, ofrecen las redes sociales; (c) Reflexión sobre los hábitos de uso de las redes sociales; (d) Exposición de las posibles sinergias entre las redes sociales y los medios de comunicación. Los cuestionarios, de 30 preguntas cada uno, se han utilizado como principal herramienta metodológica que ha posibilitado la definición de diferentes indicadores a partir de las respuestas obtenidas. En este sentido, se han incorporado preguntas abiertas y cerradas.

Las universidades participantes han sido la Faculdade Cásper Líbero (FCL) de São Paulo (Brasil), la Universidad Pontificia Bolivariana (UPB) de Medellín (Colombia) y la Universidad Autónoma de Barcelona (España). La FCL, fundada en 1947, fue la primera institución latinoamericana en poseer un curso de periodismo. La Facultad de Comunicación Social de la UPB, con más 45 años de vida, posee una acreditación internacional y otra del Ministerio de Educación Nacional de Colombia y está considerada como una de las diez mejores universidades del país. Por su parte la UAB, situada como la mejor universidad de España en el ranking "THE WUR 2016-2017", posee doce especialidades (una de ellas la de Comunicación) entre las cien mejores del mundo, según el ranking por disciplinas científicas que elabora QS a partir de algunos indicadores del QS WUR 2016-2017.

\section{Resultados}

La investigación, con un talante descriptivo, pretende aportar datos de interés para la confección de un diagnóstico general sobre el uso de las redes sociales por parte de los estudiantes de Comunicación de Brasil, Colombia y España. En su primera fase, el estudio se centró en identificar la concepción, el tipo de utilización y la forma de valoración de las redes sociales por los universitarios seleccionados. Respecto a la definición de una red social, se destaca que los estudiantes señalen básicamente aspectos positivos (cualidades y virtudes) de 
estas plataformas y que, entre los 18 conceptos más utilizados, únicamente aparezca una debilidad o elemento problemático (Adicción). También resulta interesante que la mayoría de los conceptos más mencionados aludan a aspectos vinculados con lo comunicativo: "interactividad", "inmediatez", "utilidad" o la misma palabra "comunicación". Este dato enfatiza el rol que el alumnado confiere a las redes sociales en sus intercambios y procesos comunicativos, y resalta la importancia que le confieren como instrumento de comunicación (Figura 1).

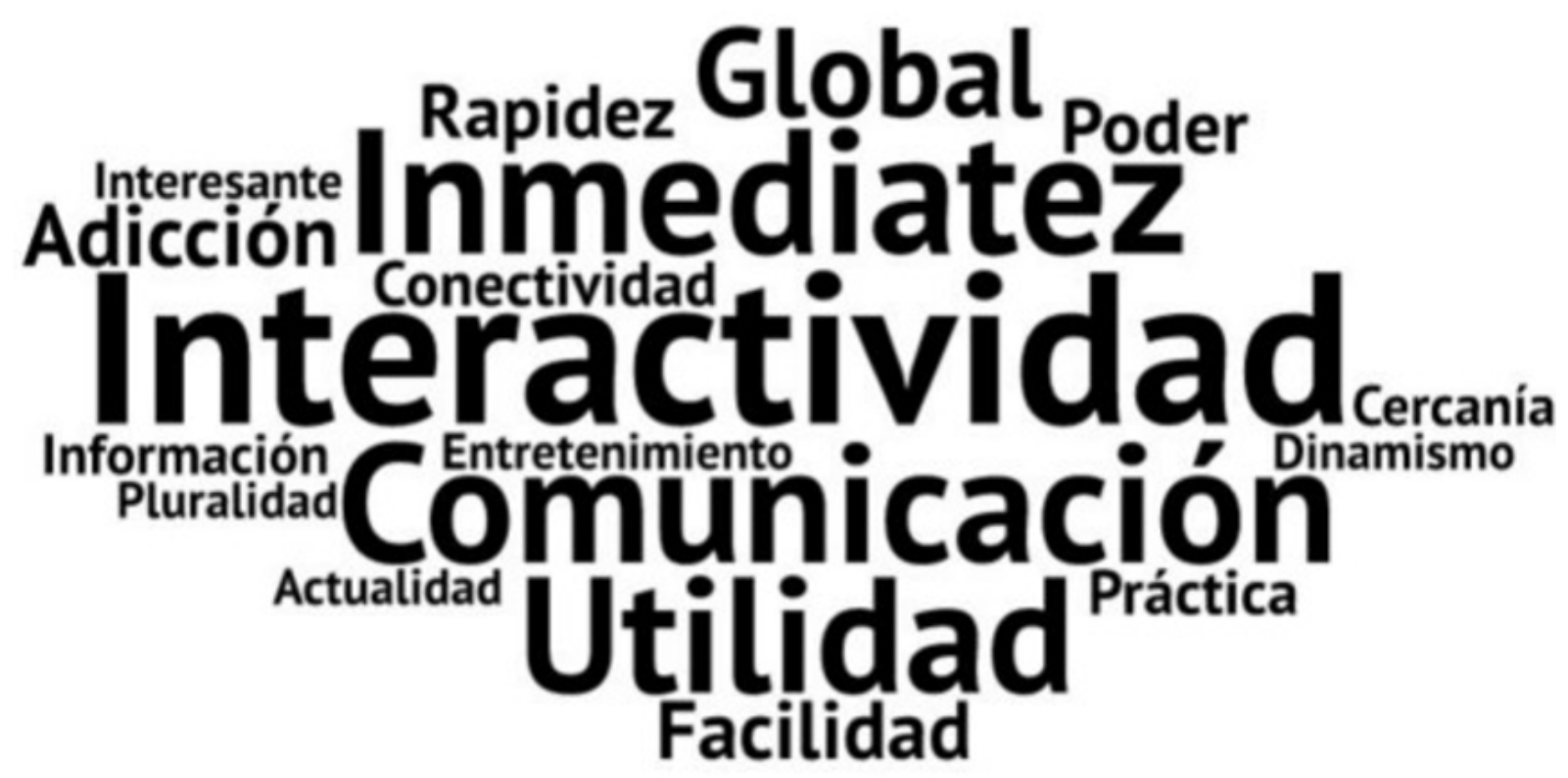

Figura 1. ¿Con qué adjetivo definirías una red social?

Fuente: Elaborada por los autores (2017).

Por otro lado, prácticamente la totalidad del alumnado tiene presencia en redes sociales (98,7\% en Brasil; 99,3\% en Colombia; y 99,6\% en España) y en los tres países, las plataformas Facebook, Twitter e Instagram son, en este orden, las que tienen mayor aceptación. Asimismo, más de un 70,0\% de los universitarios reconoce haber creado un perfil en más de una plataforma, siendo Brasil $(55,1 \%)$ y España $(53,2 \%)$ los países de la muestra que poseen un mayor porcentaje de estudiantes con presencia en tres o más plataformas. Estos resultados refuerzan la tendencia general de los últimos años (Comisión Económica para América Latina y el Caribe, 2015): Facebook se ha consolidado como una de las redes sociales más utilizadas de América Latina, alcanzando en 2014, el primer lugar (con cerca de 144.900.000 millones de usuarios), seguida de Linkedln (34.700.000), Twitter (29.153.000) y Taringa (27.600.000).

Pero las diferencias entre los tres países se tornan más evidentes al diagnosticar la edad de iniciación del estudiantado en las redes, así como al analizar el tiempo que invierten diariamente en ellas. El estudio indica que antes de los 13 años cerca de un 73,00\% de los estudiantes encuestados de Brasil y Colombia ya habían abierto perfiles en redes sociales. En España, al contrario, apenas un 32,43\%, siendo a partir de los 14 años cuando la mayoría del alumnado pasa a utilizar redes sociales.

\section{Redes sociales y comunicación}

La segunda fase del estudio interrogó a los universitarios sobre sus expectativas profesionales con el objetivo de relacionar las redes sociales con su perfil de futuros comunicadores. Los encuestados debían puntuar del 1 al 
6 (siendo 1 la opción menos valorada) las siguientes opciones: "Prensa”, "Radio", "Televisión", "Fotografía," "Internet" y "Otros". Los resultados definen tres escenarios dispares donde las preferencias del alumnado son muy diversas. La televisión (como primera opción en Colombia; y segunda en Brasil y España) es el único medio que, en cierto modo, aparece entre las preferencias profesionales del alumnado de forma general (consolidándose, en todos los casos, como la primera o la segunda elección). Internet se sitúa como la primera opción para los estudiantes brasileños, siendo la opción mejor valorada; mientras que en Colombia y España se ubica como la quinta elección entre las prioridades de los estudiantes. En conjunto, se detecta una valoración positiva de la Red que, uniendo las primeras y segundas elecciones, se acerca, en todos los casos, al 50,0\% del total (en Brasil un 62,8\%; en Colombia un 42,7\%; y en España un 46,4\%) (Tabla 1).

Partiendo de los datos anteriores, se pasó a analizar "para qué" y "cómo" los alumnos de Comunicación de estos tres países utilizan las redes sociales. Los principales usos en todos los casos, aunque con pequeñas diferencias en los porcentajes, denotan claras coincidencias. El tipo de utilización que hacen los estudiantes de estas plataformas se puede clasificar, de mayor a menor utilización, de la siguiente forma: "para informarme, "para pasar el rato (entretenimiento)" y "para buscar relaciones sociales". Curiosamente, la opción "para buscar trabajo" no obtuvo importancia en ningún país. Pero entre los tres escenarios, se detectan algunos matices: en Brasil, la opción de entretenimiento (39,7\%) superó a las de informarse $(21,8 \%)$ o entablar relaciones sociales $(24,4 \%)$. Ya en Colombia y España los resultados fueron similares (para informarse: $43,5 \%$ y $48,2 \%$, respectivamente; pasar el rato: $37,0 \%$ y $34,7 \%$; $y$ relaciones sociales: $5,1 \%$ y $2,7 \%)$.

Tabla 1. ¿En qué medio te gustaría desarrollarte como profesional?

\begin{tabular}{|c|c|c|c|c|c|c|c|}
\hline Países & & Prensa (\%) & Radio (\%) & Televisión (\%) & Fotografía (\%) & Internet (\%) & Otros (\%) \\
\hline \multirow{7}{*}{ Brasil } & 6 & 10,3 & 10,3 & 34,6 & 18,0 & 39,7 & 16,7 \\
\hline & 5 & 5,1 & 11,5 & 28,2 & 20,5 & 23,1 & 11,5 \\
\hline & 4 & 15,4 & 20,5 & 15,4 & 25,6 & 24,4 & 16,7 \\
\hline & 3 & 23,1 & 14,1 & 12,8 & 9,0 & 7,7 & 20,5 \\
\hline & 2 & 19,2 & 15,4 & 2,6 & 14,1 & 1,3 & 9,0 \\
\hline & 1 & 21,8 & 24,4 & 5,1 & 9,0 & 2,6 & 15,4 \\
\hline & $\mathrm{N} / \mathrm{C}$ & 5,1 & 3,9 & 1,3 & 3,9 & 1,3 & 10,3 \\
\hline \multirow{7}{*}{ Colombia } & & Prensa & Radio & Televisión & Fotografía & Internet & Otros \\
\hline & 6 & 26,8 & 26,8 & 29,0 & 27,5 & 13,0 & 21,7 \\
\hline & 5 & 15,2 & 31,2 & 19,6 & 20,3 & 29,7 & 13,0 \\
\hline & 3 & 15,2 & 11,6 & 9,4 & 13,0 & 16,7 & 15,2 \\
\hline & 2 & 10,1 & 7,3 & 13,8 & 9,4 & 9,4 & 6,5 \\
\hline & 1 & 10,1 & 5,8 & 8,0 & 6,5 & 3,6 & 14,5 \\
\hline & $\mathrm{N} / \mathrm{C}$ & 2,9 & 1,5 & 2,2 & 2,2 & 2,2 & 14,5 \\
\hline \multirow{7}{*}{ España } & & Prensa & Radio & Televisión & Fotografía & Internet & Otros \\
\hline & 6 & 32,9 & 24,8 & 28,8 & 20,7 & 18,0 & 8,6 \\
\hline & 5 & 28,4 & 32,9 & 25,2 & 17,6 & 28,4 & 7,2 \\
\hline & 4 & 19,8 & 15,8 & 15,8 & 19,8 & 30,6 & 11,7 \\
\hline & 3 & 7,2 & 13,1 & 14,0 & 18,5 & 14,9 & 14,4 \\
\hline & 2 & 7,7 & 9,0 & 11,7 & 13,5 & 5,9 & 12,2 \\
\hline & $\mathrm{N} / \mathrm{C}$ & 0,0 & 0,9 & 0,9 & 1,4 & 0,9 & 23,4 \\
\hline
\end{tabular}

Fuente: Elaborada por los autores (2017). 
Estos resultados están vinculados con el tipo de usuarios con los que se relacionan los estudiantes de Comunicación a través de Internet. En los tres escenarios, se destaca una utilización de las redes sociales para contactar con "amigos" (98,72\% en Brasil; 97,10\% en Colombia y 95,50\% en España). En segundo lugar, los "profesionales" serían los perfiles con los que los estudiantes mantienen una mayor interacción (35,90\%; 25,36\% y $27,93 \%$, respectivamente). Estos resultados, que sitúan a los "amigos" y a los "profesionales" como los principales destinatarios de sus interacciones, reforzarían el uso informativo y el uso social de las redes que anteriormente se señalaban como los prioritarios.

Asimismo, se indagó sobre la credibilidad que los estudiantes de comunicación confieren a los medios y a los contenidos digitales. Resulta muy interesante que, en los tres países, más de la mitad de los estudiantes (más de 55,00\% en Brasil y Colombia, y 72,00\% en España) confiera "bastante" credibilidad a los periódicos como fuentes de información dentro de una red social. Este aspecto advierte a los medios de comunicación de la importancia estratégica de distribuir sus contenidos en las diferentes plataformas de la Web 2.0. Además, la credibilidad concedida a las cabeceras digitales supera - en el nivel de "bastante" - a la que los estudiantes de comunicación otorgan a los "periodistas" (37,18\%, 47,10\% y 72,97\%, respectivamente). Únicamente, en el escenario español, el nivel de credibilidad entre "periódicos" $y$ "periodistas" es muy similar (Tabla 2).

Por otro lado, hay un claro descenso de la credibilidad conferida a otras fuentes dentro de una red social. En el caso de los políticos, se detecta una caída radical en el nivel de "bastante" credibilidad (con un 0,00\%; un 2,90\% y un $4,05 \%$, respectivamente). Sobre las páginas gubernamentales, se perciben igualmente una gran diferencia respecto a otras fuentes de información (19,23\%, 23,19\% y 43,24\%, respectivamente). El caso español, de nuevo, presenta resultados alejados de Brasil y Colombia, países donde la credibilidad que poseen las fuentes vinculadas al gobierno, dentro de una red social, es mucho menor. Dichos porcentajes invitan a reflexionar y, por ende, a desarrollar investigaciones sobre los diferentes niveles de credibilidad que los usuarios confieren a cada una de las tipologías de fuentes informativas que existen en la Red.

El estudio también preguntó a los futuros comunicadores sobre la relación entre las redes sociales y los medios de comunicación. Así, el alumnado de los tres países considera que los medios deben incorporar redes

Tabla 2. ¿Qué credibilidad confieres a estas fuentes en una red social?

\begin{tabular}{|c|c|c|c|}
\hline & Brasil (\%) & Colombia (\%) & España (\%) \\
\hline \multicolumn{4}{|l|}{ Periódicos } \\
\hline Ninguna credibilidad & 0,00 & 0,00 & 0,00 \\
\hline Poca credibilidad & 1,28 & 5,80 & 0,00 \\
\hline Alguna credibilidad & 25,64 & 19,57 & 11,26 \\
\hline Bastante credibilidad & 55,13 & 55,07 & 72,07 \\
\hline Toda la credibilidad & 14,10 & 15,22 & 14,86 \\
\hline $\mathrm{N} / \mathrm{C}$ & 3,85 & 4,35 & 1,80 \\
\hline \multicolumn{4}{|l|}{ Periodistas } \\
\hline Ninguna credibilidad & 1,28 & 0,00 & 0,00 \\
\hline Poca credibilidad & 2,56 & 7,97 & 0,00 \\
\hline Alguna credibilidad & 44,87 & 32,61 & 13,06 \\
\hline Bastante credibilidad & 37,18 & 47,10 & 72,97 \\
\hline Toda la credibilidad & 10,26 & 9,42 & 12,16 \\
\hline $\mathrm{N} / \mathrm{C}$ & 3,85 & 2,90 & 1,80 \\
\hline
\end{tabular}

Fuente: Elaborada por los autores (2017). 
sociales a sus plataformas informativas (73,08\% en Brasil, $68,84 \%$ en Colombia y $83,78 \%$ en España). Este dato se refuerza al verificar que, en todos los casos, alrededor del 50,00\% de los estudiantes están agregados a la red social de algún medio (con un 60,26\%, un 49,28\% y un 49,10\%, respectivamente). Además, en los tres países se constata la preferencia de la mitad o más de los estudiantes por una red social frente a un medio de comunicación online (Tabla 3).

Este aspecto puede relacionarse con los comentarios que los estudiantes realizan, en las preguntas abiertas, al identificar una fortaleza y una debilidad de las redes sociales. En su mayoría, los testimonios aluden al potencial comunicativo de estas plataformas como espacios virtuales donde acceder a un volumen de información más completo y variado en su procedencia y en sus atributos. Además, se detecta una referencia recurrente a los riesgos derivados del exceso de información, la circulación de informaciones sin contrastar y la falta de privacidad (Cuadro 1).

Este conjunto de reflexiones están vinculadas a la pregunta decisiva de la investigación que interrogó al alumnado sobre si consideraban una red social como una plataforma donde podrían encontrar trabajo. La gran mayoría de estudiantes respondió afirmativamente (Brasil, 91,03\%; Colombia, 89,86\%; España, 89,64\%), aspecto que enfatiza su concepción de estas plataformas como escenarios válidos para su desempeño profesional. Inversamente, las respuestas también son similares sobre el carácter efímero de las redes sociales. Solo la minoría de estudiantes $(11,54 \%, 13,04 \%$ y 13,96\%, respectivamente) considera las redes sociales una "moda pasajera".

Tabla 3. ¿Qué prefieres una red social o un medio de comunicación online?

\begin{tabular}{lccc}
\hline & Brasil (\%) & Colombia (\%) & España (\%) \\
\hline Red Social & 69,23 & 50,00 & 53,15 \\
Medio de comunicación online & 21,79 & 40,58 & 43,24 \\
N/C & 8,97 & 9,42 & 3,60 \\
\hline
\end{tabular}

Fuente: Elaborada por los autores (2017).

Cuadro 1. ¿Con qué adjetivo definirías una red social?

\begin{tabular}{ll}
\hline Fortaleza & Debilidad \\
\hline "Llegar a muchas personas". & "Puede generar dependencia, a veces la información puede ser del \\
& todo no veraz y la identidad de algunas personas puede ser mentira".
\end{tabular}

"Actualización continua de la información".

"Lograr promocionar la imagen de una empresa".

"Estar constantemente actualizado".

"Hay especialistas frente a diversos temas".
"La sobreabundancia de contenidos sin interés informativo, el hecho de que no haya un control sobre los comentarios y la poca privacidad que ofrecen".

"Falsedad, exceso de información y posibilidad de mentir y crear falsedades".

"Poca credibilidad".

"Se confía demasiado en lo que dicen los usuarios, y no siempre es bueno". 


\section{Conclusión}

Los resultados del estudio permiten concluir que los estudiantes de Comunicación de los países analizados presentan una concepción común de las redes sociales en cuanto a su definición, su importancia y su utilidad, más allá de las diferencias socioeconómicas que existen entre estos tres escenarios. Se verifica, de este modo, la hipótesis 1 del presente artículo. Salvo en algunas cuestiones puntuales, se detecta que los universitarios del ámbito de la Comunicación confieren la misma importancia a las redes sociales. Es posible afirmar que el grado de presencia y de utilización de las redes sociales no posee una relación intrínseca con el nivel de desarrollo o con la coyuntura socioeconómica de los países. Las redes sociales se consolidan como plataformas de vital importancia para los jóvenes usuarios de la Red, quienes incluso poseen ecosistemas digitales integrados, en la mayoría de casos, por más de una red.

Relacionado con ello, y validando la hipótesis 2 del trabajo, es posible afirmar que la concepción de estas plataformas por el estudiantado de Comunicación responde, de forma mayoritaria, a cualidades y características positivas, obviando los elementos negativos relacionados con este tipo de plataformas. Este aspecto introduce la necesidad de fomentar una alfabetización digital y mediática de los usuarios más jóvenes que no puede detenerse en el simple uso y dominio instrumental o técnico de las herramientas digitales. Como la utilización de las redes sociales entre este estudiantado se centra prioritariamente en ocio y entretenimiento, sería necesario potenciar la mirada crítica de los usuarios dotando los estudiantes de la capacidad de analizar, contrastar y construir contenidos a partir de los recursos e instrumentos online. Finalmente, la hipótesis 3 no queda validada ya que se ha detectado que los universitarios sí valoran las posibilidades profesionales que les ofrecen las redes sociales. Estos consideran como una opción válida y real la posibilidad de desarrollarse como profesionales de la Comunicación en las redes, pero no se observa un conocimiento de los diferentes escenarios profesionales que éstas les ofrecen. Además, se plantea la necesidad de investigaciones sobre la credibilidad que los usuarios más jóvenes confieren a las fuentes de información online, la importancia de analizar las habilidades y las competencias del comunicador digital en una sociedad-red, la pertinencia de reflexionar sobre los nuevos perfiles profesionales que aparecen en el ámbito comunicativo a raíz del desarrollo de las redes sociales, entre otros.

\section{Colaboradores}

El diseño y la realización del estudio fueron coordinados por S. Tejedor. Todos los autores han contribuido en todas las etapas de la investigación, explotación y análisis de datos y redacción final.

\section{Referencias}

Associação Nacional de Jornais. A Indústria Jornalística: Cenários. Brasília, 2015. Disponível em: <http://www.anj.org.br/ home-teste/a-industria-jornalistica>. Acesso em: 20 jul. 2017.

Baek, K. et al. The link that bind: Uncovering novel motivations for linking on Facebook. Computers in Human Behavior, v. 27, n. 6, p. 2243-2248, 2011. https://doi.org/10.1016/j.chb.2011. 07.003

Brasil. Ministério da Ciência, Tecnologia, Inovações e Comunicações. Dados do setor de Comunicações. Brasília: MCTIC, 2017. Disponível em: <http://sistema.mc.gov.br/DSCOM/ view/Informacoes.php >. Acesso em: 6 mar. 2018.

Bulut, Z. A.; Doğan, O. The ABCD typology: Profile and motivations of Turkish social network sites users. Computers in
Human Behavior, v. 67, n. 2, p. 73-83, 2017. https://doi.org/10. 1016/j.chb.2016.10.021

Cáceres, M. D.; Morales, E. Juventud hiperconectada. Comunicación y sociabilidad virtual. Estudio de caso sobre desconexión de medios durante 24 horas en jóvenes universitarios españoles. Anuario electrónico de estudios en Comunicación Social."Disertaciones", v. 7, n. 2, p. 160-177, 2014. Disponible en: <http://erevistas.saber.ula.ve/index.php/ Disertaciones/article/view/4687>. Acceso en: 23 ene. 2017.

Carbonell, X. et al. Adicción a Internet y Móvil: una revisión de estudios empíricos españoles. Papeles del Psicólogo, v. 33, n. 2, p. 82-89, 2012. Disponible en: <http://www. papelesdelpsicologo.es/resumen?pii=2096>. Acceso en: 12 ene. 2017. 
Colombia. Ministerio de Tecnologías de la Información y las Comunicaciones. Plan Vive Digital. Bogotá: Mintic, 2014. Disponible en: <http://www.mintic.gov.co/portal/ vivedigital/612/w3-channel.html>. Acceso en: 11 sept. 2016.

Comisión Económica para América Latina y el Caribe. La nueva revolución digital: de la Internet del consumo a la Internet de la producción. Santiago: Cepal, 2015. Disponible en: <http:// repositorio.cepal.org/bitstream/handle/11362/38604/4/ S1600780_es.pdf>. Acceso en: 26 nov. 2016.

Crovi, D.; López, R. Tejiendo voces. Jóvenes universitarios opinan sobre la apropiación de Internet en la vida académica. Chasqui: Revista Latinoamericana de Comunicación, n. 117, p. 3-10, 2012.

Espinar-Ruiz, E.; González-Río, M. J. Uso de Internet y prácticas políticas de los jóvenes españoles. Convergencia Revista de Ciencias Sociales, n. 69, p. 13-38, 2015. Disponible en: <http:// convergencia.uaemex.mx/article/view/3632>. Acceso en: 12 ene. 2017.

Gobernación De Antioquia. Antioquia Digital. Metaportal, Antioquia, 2013. Disponible en: <http://www.antioquiadigital. edu.co>. Acceso: 24 mar. 2017.

Herrero Curiel, E. La credibilidad de las redes sociales en el ámbito periodístico. TransInformação, v. 27, n. 2, p. 165-171, 2015. http://dx.doi.org/10.1590/0103-37862015000200006

Interactive Advertising Bureau - Spain; Elogia. Estudio Anual de Redes Sociales 2017. Madrid: IAB, 2017. Disponible en: <http://iabspain.es/wp-content/uploads/iab_estudioredes sociales_2017_vreducida.pdf>. Acceso en: 23 mayo. 2017.

Instituto Brasileiro de Opinião Pública e Estatística. Pesquisa Brasileira de Mídia. Rio de Janeiro: IBOPE, 2016. Disponível em: <http://www.secom.gov.br/atuacao/pesquisa/lista-depesquisas-quantitativas-e-qualitativas-de-contratos-atuais/ pesquisa-brasileira-de-midia-pbm-2016.pdf/view>. Acesso em: 23 maio 2017.

Kemp, S. Digital in 2017: Global overview. New York: We are Social, 2017. Available from: <https://wearesocial.com/ special-reports/digital-in-2017-global-overview>. Cited: Feb. 18, 2017.

Masip, P. et al. News and social networks: Audience behavior. El profesional de la información, v. 24, n. 4, p. 363-370, 2015. https://doi.org/10.3145/epi.2015.jul.02

Matsa, K. E.; Mitchell, A. 8 keys takeaways about Social Media and news. Washington: Pew Research Center, 2014. Available from: <http://www.journalism.org/2014/03/26/8-key-takeawaysabout-social-media-and-news/> . Cited: Jan, 302017.

Medellín es la ciudad líder en conectividad en Latinoamérica. Caracol Radio, Bogotá, 2015. Disponible en: <http://caracol. com.co/emisora/2015/10/02/medellin/1443790838_413265. html>. Acceso en: 15 mayo 2016.

Muñoz-Rivas, M.; Navarro, M.; Ortega, N. Patrones de uso de Internet en población universitaria española. Adicciones, v. 15, n. 2, p. 137-144, 2003. https://doi.org/10.20882/adicciones.437

Papacharissi, Z.; Mendelson, A. Toward a new(er) sociability: Uses, gratifications and social capital on Facebook. In: Papathanassopoulos, S. (Ed.). Media perspectives for the 21st century. New York: Routledge, 2011. p. 212-230.

Pérez Tornero, J. M.; Tejedor, S. (Ed.). Ideas para aprender a aprender. Manual de innovación educativa y tecnología. Barcelona: Editorial UOC, 2016.

Purcell, K. et al. Understanding the participatory news consumer: How internet and cell phone users have turned news into a social experience. Washington: The Pew Internet and American Life Project, 2010. Available from: <http://www. pewinternet.org/files/old-media/Files/Reports/2010/PIP_ Understanding_the_Participatory_News_Consumer.pdf $>$. Cited: Jan. 31, 2017.

Ruano, L.; Congote, E.; Torres, A. Comunicación e interacción por el uso de dispositivos tecnológicos y redes sociales virtuales en estudiantes universitarios. Revista Ibérica de Sistemas e Tecnologias de Informação, n. 19, p. 15-31, 2016. https://doi.org/10.17013/risti.19.15-31

Ruiz de Miguel, C. Perfil de uso del teléfono móvil e Internet en una muestra de universitarios españoles ¿Usan o abusan? Bordón: Revista de pedagogía, v. 68, n. 3, p. 131-145, 2016. https://doi.org/10.13042/Bordon.2016.68307

Salaverría, R. Redefinir al comunicador. El profesional de la información, v. 25, n. 2, p. 163-167, 2016. https://doi. org/10.3145/epi.2016.mar.02

Unión Internacional de Telecomunicaciones. Medición de la Sociedad de la Información. Ginebra: UIT, 2012. Resumen ejecutivo. Disponible en: <https://www.itu.int/dms_pub/ itu-d/opb/ind/D-IND-ICTOI-2012-SUM-PDF-S.pdf>. Acceso: 4 dic. 2016.

Whiting, A.; Williams, D. Why people use social media: a uses and gratifications approach. Qualitative Market Research: An International Journal, v. 16, n. 4, p. 362-369, 2013. https://doi. org/10.1108/QMR-06-2013-0041 